\title{
Surface and capillary transitions in an associating binary mixture model
}

\author{
J. M. Romero-Enrique, ${ }^{1,2, *}$ L. F. Rull, ${ }^{2}$ and U. Marini Bettolo Marconi ${ }^{3}$ \\ ${ }^{1}$ Department of Mathematics, Imperial College of Science, Technology and Medicine, 180 Queen's Gate, \\ London SW7 2BZ, United Kingdom \\ ${ }^{2}$ Departamento de Física Atómica, Molecular y Nuclear, Area de Física Teórica, Universidad de Sevilla, \\ Apartado de Correos 1065, 41080 Sevilla, Spain \\ ${ }^{3}$ Dipartimento di Fisica and Istituto Nazionale di Fisica della Materia, via Madonna delle Carceri, \\ Università di Camerino, 62032 Camerino, Italy \\ (Received 3 December 2002; published 11 April 2003)
}

\begin{abstract}
We investigate the phase diagram of a two-component associating fluid mixture in the presence of selectively adsorbing substrates. The mixture is characterized by a bulk phase diagram that displays peculiar features such as closed loops of immiscibility. The presence of the substrates may interfere with the physical mechanism involved in the appearance of these phase diagrams, leading to an enhanced tendency to phase separate below the lower critical solution point. Three different cases are considered: a planar solid surface in contact with a bulk fluid, while the other two represent two models of porous systems, namely, a slit and an array on infinitely long parallel cylinders. We confirm that surface transitions, as well as capillary transitions for a large surface area to volume ratio, are stabilized in the one-phase region. Applicability of our results to experiments reported in the literature is discussed.
\end{abstract}

DOI: 10.1103/PhysRevE.67.041502

PACS number(s): 64.75. $+\mathrm{g}$, 05.70.Np, 68.08.Bc, 68.35.Rh

\section{INTRODUCTION}

In the last few years there has been a considerable progress in the understanding of the physics of fluids in restricted geometries [1]. The properties of liquids or gases confined in narrow pores appear different from those in the bulk. A common trend of these systems is the fact that condensation occurs at pressures different from the saturation bulk pressure and the critical temperature for phase separation is generally lower than the corresponding bulk temperature. According to Nakanishi and Fisher [2-5], an incompressible fluid mixture (with isotropic interactions) under the effect of confinement displays a reduced tendency toward phase separation at a fixed temperature, because the effective attractive forces result weaker within a pore. The confinement also determines an effective dimensional reduction thus enhancing fluctuation effects. In addition, the presence of adsorbing walls may induce a surface transition in a subcritical fluid, even when this is in a single-phase region in the bulk. Such a behavior, which is now well understood by means of a mean-field approach [6-8] is due essentially to the reduction of the contribution to the free energy from the cohesive fluid-fluid forces caused by the presence of the confining walls. There exists, however, a class of nonsimple fluid mixtures for which the situation is different: these are the strongly associating mixtures. Strong directional attractive interactions such as hydrogen bonding or charge transfer complexing between molecules affect dramatically the properties of the fluid or solid. An interesting phenomenon that occurs in such a mixture is the reentrant miscibility: the mixture is completely miscible at low temperatures, up to a temperature where it separates into two liquid phases. As the

\footnotetext{
*Corresponding author. Email address: jose.enrique@ imperial.ac.uk
}

temperature is further increased, the complete miscibility reappears. The nicotine-water mixture is a paradigm for such a behavior [9]. The temperature-composition phase diagram shows closed loops of immiscibility, characterized by the existence of a lower and an upper critical solution point (LCSP and UCSP, respectively). Hirschfelder et al. [10] proposed a mechanism to explain the reentrant miscibility. The essential ingredient is the existence of highly directional strong attraction (as the hydrogen bond) between the unlike particles. The dispersive (isotropic) forces between these particles are assumed to be so weak as to favor segregation of the species. At low temperatures, the energy favors complete mixing. However, as the temperature increases, the energy decrement due to the formation of directional bonds competes with the decrease of orientational entropy. As a consequence, the system phase separates when the temperature raises. This mechanism has been confirmed by theoretical studies on lattice [11-13] and continuous models [14], as well as in recent computer simulation studies $[15,16]$.

How the presence of substrates or the confinement in a pore affect the behavior of these mixtures (if in a manner similar to that of simple fluids, or if on the contrary these mixtures are peculiar) is an issue of great practical and theoretical interest. The presence of selectively adsorbing substrates can interfere with the formation of bulk hydrogen bonding, leading to a stabilization of the two-phase region with respect to the completely miscible phase. Computer simulation studies on confined lattice models [17] show the appearance of surface transitions in a range of temperatures below the LCSP. The same mechanism has been invoked to explain some experiments $[18,19]$ that seemed to contradict the Nakanishi-Fisher picture.

In the present paper we shall study a lattice model of associating binary mixtures, introduced few years ago by Lin and Taylor $[20,21]$ and reconsidered recently by the present authors [22-25]. In spite of its idealized nature, the model has the merit of allowing for an explicit analysis of its non- 


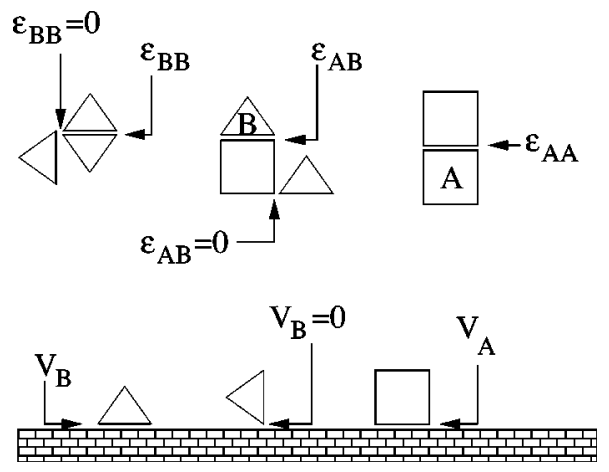

FIG. 1. Schematic representation of the $A-B$ LT model in the two-dimensional square lattice (the underlying lattice is not shown for clarity). Relevant fluid-fluid and fluid-substrate interactions are also highlighted. (See text for explanation.)

trivial phase diagram. As an instance, the study of its bulk properties predicts the existence of closed loops of immiscibility under constant pressure conditions. This analysis will be extended to the behavior of the mixture in presence of substrates and under confinement.

The structure of the paper is organized as follows. In Sec. II we briefly present the model and write explicitly the equations for the order parameter profiles. Section III is devoted to analyze in detail the wetting behavior of the mixture in the presence of adsorbing substrates. In Sec. IV we study the capillary behavior of the same system when confined between two parallel plates (a slit) and in Sec. V we consider the same system confined in a network of very narrow cylinders. Capillary transitions (and surface transitions for the slab geometry) will be analyzed in terms of the physical parameters of the system. Finally, in Sec. VI we present our conclusions.

\section{THE MODEL}

Some years ago, Lin and Taylor proposed a lattice model to describe a binary mixture, in which the two unlike species can form highly directional bonds. In more detail, the LinTaylor (LT) model $[20,21]$ is an $A-B$ binary mixture lattice model, defined on a generic $D$-dimensional lattice of coordination number $\nu$. The $A$ particles are allowed to singly occupy any of the lattice cells. Every cell contains $\nu$ equal subcells, one for each face of the mother cell. Again, each subcell can host at most one $B$ particle, provided the mother cell is not occupied by an $A$ particle. In addition to the repulsive interactions (which determine the previous occupation rules), one includes short-ranged interactions (see Fig. 1). The interaction strength between a pair of nearestneighbor $A$ particles is $\epsilon_{A A}, \epsilon_{A B}$ between unlike species and $\epsilon_{B B}$ between $B-B$ particles. All these interactions are nonvanishing only if the particles share a face.

The bulk properties, as well as the global phase diagram, have been exhaustively analyzed in previous work [22-25]. One of the main conclusions of such studies is that the appearance of closed loops of immiscibility occurs when the interaction between $A-A$ and $A-B$ pairs are attractive ( $\epsilon_{A A}$ $\left.<0, \epsilon_{A B}<0\right)$, and $\epsilon_{A B} \lesssim\left[\epsilon_{A A}+\min \left(\epsilon_{B B}, 0\right)\right] / 2$. The latter con- dition is in complete agreement with the mechanism proposed by Hirschfelder, Stevenson, and Eyring [10] for the reentrant miscibility. In this work, we assume the bulk fluidfluid parameters to have values: $\epsilon_{A A}=-1$ (it defines the energy scale), $\epsilon_{A B}=-0.65$, and $\epsilon_{B B}=0$, so that we are in the regime where closed loops of immiscibility appear.

The presence of substrates introduces new interactions (see Fig. 1). We shall assume that the substrates do not affect the fluid-fluid interactions, i.e., there is no surface enhancement. The interaction strength between an $A$ particle and the substrate is $V_{A}$, if the particle is in a cell in contact with the substrate, and zero otherwise. The interaction between the $B$ particles and the substrate is orientationally dependent, i.e., its strength $V_{B}$ is nonvanishing only if the face of the $B$ particle touches the substrate. The form of the substrate-fluid interactions are consistent with the bulk interactions. Hereafter, the substrate-fluid interactions will be assumed as attractive, i.e., $V_{A} \leqslant 0$ and $V_{B} \leqslant 0$. For absolute values of $V_{B}$ large enough, the substrate- $B$ interactions compete with the bulk $A-B$ interactions, so that one expect this type of substrate to enhance the demixing tendency for temperatures lower than the corresponding LCSP.

To study the LT model in presence of substrates, we consider the grand-canonical ensemble, where the system is in thermal and chemical equilibrium with a reservoir at fixed temperature $T$ and chemical potentials $\mu_{A}$ and $\mu_{B}$. The activities $z_{A}$ and $z_{B}$ are defined via $z_{A} \equiv \exp \left(\beta \mu_{A}\right)$ and $z_{B}$ $\equiv \exp \left(\beta \mu_{B}\right)$, where $\beta=1 / k_{B} T$. After tracing over all configurations of the $B$ particles, the system results to be isomorphic to a monocomponent lattice gas, with renormalized interactions and chemical potential. The details on this procedure can be found in Refs. [21,23,25]. The grand-canonical partition function $\Xi$ of the LT model can be written in terms of an equivalent Ising model as

$$
\begin{aligned}
\Xi= & \left(1+2 z_{B}+z_{B}^{2} e^{\left.-\beta \epsilon_{B B}\right)^{\nu N / 2}} \exp \left[N\left(H-\frac{\nu K}{2}\right)\right]\right. \\
& \times\left(\frac{1+z_{B} e^{-\beta V_{B}}}{\sqrt{1+2 z_{B}+z_{B}^{2} e^{-\beta \epsilon_{B B}}}}\right)^{\nu_{\perp} N_{b}} \\
& \times \exp \left[N_{b}\left(\Delta H_{1}+\frac{\nu_{\perp} K}{2}\right)\right] Z_{D, \nu}^{I s i n g}\left(K, H, \Delta H_{1}\right),
\end{aligned}
$$

where $N$ is the total number of lattice cells, $N_{b}$ is the number of cells adjacent to the substrate, $\nu_{\perp}$ is the number of nearest neighbors in the direction perpendicular to the substrate; $\nu_{\|}$ is defined as the number of nearest-neighbor cells in directions parallel to the substrate. Obviously, $\nu=\nu_{\|}+2 \nu_{\perp}$. Finally, $Z_{D, \nu}^{I s i n g}\left(K, H, \Delta H_{1}\right)$ is the canonical partition function of the Ising model on the same lattice. Let us name $K$ the effective coupling constant, $H$ the effective bulk magnetic field, and $\Delta H_{1}$ the effective surface magnetic field acting only on the boundary sites,

$$
K=-\frac{\beta \epsilon_{A A}}{4}+\frac{1}{2} \ln \left(\frac{\sqrt{1+2 z_{B}+z_{B}^{2} e^{-\beta \epsilon_{B B}}}}{1+z_{B} e^{-\beta \epsilon_{A B}}}\right),
$$




$$
\begin{gathered}
H=\frac{1}{2} \ln z_{A}-\frac{\nu \beta \epsilon_{A A}}{4}-\frac{\nu}{4} \ln \left(1+2 z_{B}+z_{B}^{2} e^{\left.-\beta \epsilon_{B B}\right),}\right. \\
\Delta H_{1}=\nu_{\perp}\left[\frac{1}{2} \ln \left(\frac{\sqrt{1+2 z_{B}+z_{B}^{2} e^{-\beta \epsilon_{B B}}}}{1+z_{B} e^{-\beta V_{B}}}\right)+\frac{\beta}{4}\left(\epsilon_{A A}-\frac{2 V_{A}}{\nu_{\perp}}\right)\right] .
\end{gathered}
$$

All equilibrium properties can be obtained from the knowledge of the partition function Eq. (1) by using standard thermodynamical relationships. In particular, the bulk pressure is obtained as

$$
\begin{aligned}
p= & \lim _{V \rightarrow \infty} k_{B} T \frac{\ln \Xi}{V}=\frac{k_{B} T}{v_{0}}\left[\frac{\nu}{2} \ln \left(1+2 z_{B}+z_{B}^{2} e^{-\beta \epsilon_{B B}}\right)-\frac{\nu K}{2}\right. \\
& \left.+H+\lim _{N \rightarrow \infty} \frac{1}{N} \ln Z_{D, \nu}^{I s i n g}\right],
\end{aligned}
$$

where $v_{0}$ is the volume of lattice cell, and $N \equiv V / v_{0}$. The mole fraction $X_{A}(i)$ profile is obtained as

$$
X_{A}(i) \equiv \frac{n_{A}(i)}{n_{A}(i)+\sum_{s=1}^{\nu} n_{B}(i, s)},
$$

where $n_{A}(i)$, the $A$-particle density in the cell $i$, is defined in terms of the local magnetization $m_{i}$ as

$$
n_{A}(i)=\frac{1+m_{i}}{2 v_{0}}
$$

and $n_{B}(i, s)$, the $B$ particle density in the subcell $s$ of the cell $i$, is

$$
n_{B}(i, s)=\frac{1-m_{i}}{2 v_{0}} \frac{z_{B} e^{-\beta V_{B}}}{1+z_{B} e^{-\beta V_{B}}},
$$

if the subcell is by the substrate (see Fig. 1). Otherwise, $n_{B}(i, s)$ is defined as

$$
\begin{aligned}
n_{B}(i, s)= & \frac{1}{4 v_{0}}\left[\frac{z_{B} e^{-\beta \epsilon_{A B}}}{1+z_{B} e^{-\beta \epsilon_{A B}}}\left(1-m_{i}+m_{j}-\left\langle s_{i} s_{j}\right\rangle\right)\right. \\
& \left.+\frac{z_{B}+z_{B}^{2} e^{-\beta \epsilon_{B B}}}{1+2 z_{B}+z_{B}^{2} e^{-\beta \epsilon_{B B}}}\left(1-m_{i}-m_{j}+\left\langle s_{i} s_{j}\right\rangle\right)\right],
\end{aligned}
$$

where $\left\langle s_{i} s_{j}\right\rangle$ is the two-spin correlation function between the magnetization on the cell $i$ and its nearest-neighbor $j$ that it is associated to the subcell $s$. It is easy to see that in the homogeneous case these equations reduce to the expressions given in Refs. [23,25].

The equivalence between the LT model and the Ising model, allows one to obtain the surface behavior of the mixture from the knowledge of the behavior of the latter. How- ever, such a mapping, is not at all trivial and may lead in some cases to unexpected results.

\section{WETTING TRANSITIONS IN THE LT MODEL}

Let us consider an $A-B$ binary mixture in the presence of a planar, infinite, and structureless substrate. Such a substrate confines the fluid to the half-space $z \geqslant 0$. When the fluid (e.g., the $A$-particle rich phase, $\alpha$ ) is at bulk coexistence, the substrate can promote the formation of drops of the opposite coexisting phase (the $B$-particle rich phase $\beta$ ) on it, characterized by a contact angle $\theta$. When $\theta=0$, the drops spread over the substrate, i.e., the phase $\beta$ wets completely the $\alpha$-substrate interface. A finite value of $\theta$, instead, corresponds to a partial wetting situation. The crossover between a partial to a complete wetting regime is called the wetting transition [26]. The surface tensions involved in the problem ( $\sigma_{\alpha s}, \sigma_{\beta s}$ and $\sigma_{\alpha \beta}$, corresponding to the $\alpha$-substrate, $\beta$-substrate, and $\alpha-\beta$ interfaces, respectively) are related to the contact angle via the Young equation [27]

$$
\sigma_{\alpha s}=\sigma_{\beta s}+\sigma_{\alpha \beta} \cos \theta
$$

Thermodynamically the surface tensions correspond to the excess grand-canonical-free energy per interfacial unit area (respect to the bulk) [27]. Since both $\sigma_{\alpha \beta}$ and $\sigma_{\beta s}$ have an analytical behavior at this thermodynamic state, the wetting transition is a (surface) thermodynamic phase transition, as it can be seen from Eq. (10) and the dependence of $\theta$ on the temperature. As usual, appropiate order parameters corresponding to the wetting transition can be defined as first derivatives of the surface tension respect to thermodynamic fields. In simple fluids, the excess number of particles per interfacial unit area or adsorption $\Gamma$ [that corresponds to $-\left(\partial \sigma_{\alpha s} / \partial \mu\right)_{T}, \mu$ being the chemical potential] is the natural order parameter choice. In mixtures, other choices are available, e.g., the volume integrated excess mole fraction per interfacial unit area. Microscopically, the wetting transition manifests itself as the growth of a macroscopically thick $\beta$ layer intruding in the $\alpha$-substrate interface composition profile. Three different types of wetting transition may arise. If the layer width jumps suddenly from a microscopically finite value, the wetting transition is of first order, and an off-coexistence surface transition (prewetting) is associated to it. If the layer width diverges continuously, the wetting transition is named critical. Finally, the wetting transition can occur as the accumulation point of an infinite sequence of first-order transitions in the layering wetting case (these transitions extend to the off-coexistence region). The conditions for such a transition depend subtly on the fluid-fluid and substrate-fluid interactions, and interfacial fluctuations can play an important role [28].

In the LT model two-phase coexistence occurs when $H$ $=0$ and $K>K_{c}$, where $K_{c}$ is the lattice dependent Ising critical coupling. Moreover, the wetting behavior is controlled by the ratio $\Delta H_{1} / \nu_{\perp} K$, that is the ratio between the surface energy and the loss of bulk energy due to the presence of the surface. If such a parameter is positive the substrate favors the $\alpha$ phase to intrude between the substrate and 

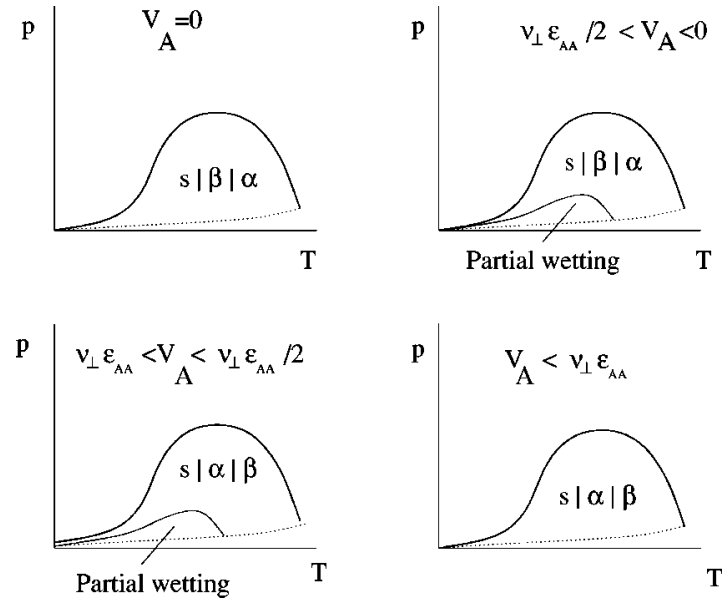

FIG. 2. Qualitative wetting phase diagrams for the LT model for $V_{B}=0$. Thick solid lines correspond to critical lines, dotted lines to the $A$-pure gas-liquid coexistence lines, and thin solid lines to the wetting transition lines. The symbol $s|\alpha| \beta(s|\beta| \alpha)$ means the bulk conditions where there exist wetting of the $\beta$-substrate ( $\alpha$-substrate) interface by the $\alpha(\beta)$ phase, respectively (see text). The bulk conditions under which there is partial wetting are also shown.

the $\beta$ phase. On the other hand, if $\Delta H_{1} / \nu_{\perp} K<0$, the phase $\beta$ intrudes between the substrate and the phase $\alpha$. We shall focus on the coexistence side $H=0^{+}\left(H=0^{-}\right)$for $\Delta H_{1}$ $<0\left(\Delta H_{1}>0\right)$, respectively (otherwise, only partial wetting can occur). If the absolute value of the parameter is greater than one $\left(\left|\Delta H_{1} / \nu_{\perp} K\right|>1\right)$, then all coexistence surface corresponds to a complete wetting situation. If such a condition is not fulfilled, one will observe a partial wetting situation up to a value $K_{w}$ (dependent on $\left|\Delta H_{1} / \nu_{\perp} K\right|$ ) and beyond it a complete wetting situation up to $K=K_{c}$.

Let us consider first the case $V_{B}=0$, when $\Delta H_{1}$ depends only on $T$, regardless the value of $z_{B}$. Moreover, the sign of $\Delta H_{1}$ is uniquely determined by the difference between $V_{A}$ and $\nu_{\perp} \epsilon_{A A} / 2$ over the whole temperature range. One can envisage various cases (see Fig. 2): if $V_{A}=0$, the coexistence corresponds to a situation of complete wetting of the interface substrate- $\alpha$ phase by phase $\beta$, denoted by the symbol $s|\beta| \alpha$. If $\nu_{\perp} \epsilon_{A A} / 2<V_{A}<0$, there exists a region of complete wetting $s|\beta| \alpha$ and a lower pressure region of partial wetting. As $V_{A}$ decreases, the region of complete wetting $s|\beta| \alpha$ shrinks and eventually disappears for $V_{A}=\nu_{\perp} \epsilon_{A A} / 2$, because $\Delta H_{1} \equiv 0$. For $V_{A}<\nu_{\perp} \epsilon_{A A} / 2$, the sign of $\Delta H_{1}$ changes and a region of complete wetting of the interface substrate-phase $\beta$ by phase $\alpha$ appears $(s|\alpha| \beta)$. As $V_{A}$ increases, the region of partial wetting reduces and disappears for $V_{A} \geqslant \nu_{\perp} \epsilon_{A A}$.

When $V_{B} \neq 0$, it is possible that in correspondence with different state points, the substrate favors either the $\beta$ or the $\alpha$ phase. To study such an effect it is necessary to locate the coexistence states, where $\Delta H_{1}=0$. Since $V_{B}$ and $\epsilon_{A B}$ are negative, for a temperature $T$ all the coexistence states with pressure $p$ larger than $p_{n w}(T) \equiv p\left(T, H=0, \Delta H_{1}=0\right)$ correspond to $\Delta H_{1}>0$, whereas states with lower pressures correspond to $\Delta H_{1}<0$. It is easy to prove from Eq. (4) that a necessary condition for the existence of this line is that $V_{B}$

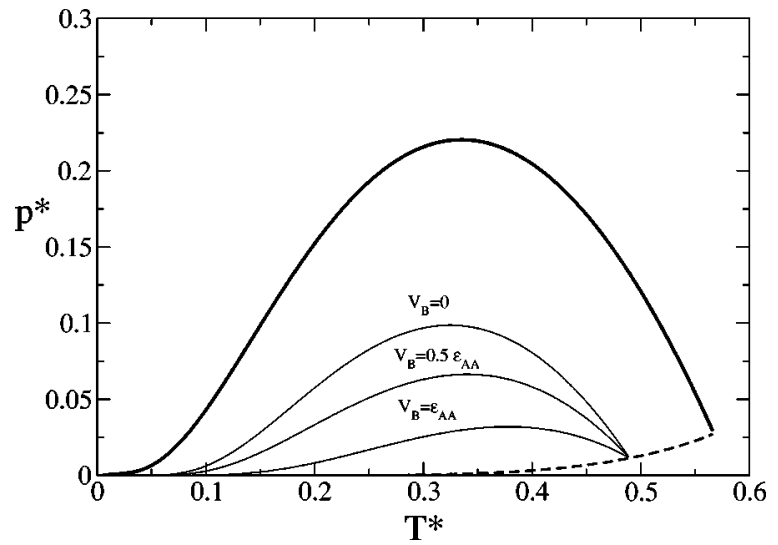

FIG. 3. Wetting phase diagram for the square lattice LT model. The bulk parameters are fixed to $\epsilon_{A B}=0.65 \epsilon_{A A}<0, \epsilon_{B B}=0$, and $V_{A}=0.25 \epsilon_{A A}$. The thick solid line corresponds to the bulk critical line, and the thick dashed line to the liquid-vapor transition line of the $A$-pure fluid. The thin solid lines correspond to the wetting transition lines for different values of $V_{B}$. Hereafter, the reduced units are defined via $T^{*} \equiv k_{B} T /\left|\epsilon_{A A}\right|$ and $p^{*} \equiv p v_{0} /\left|\epsilon_{A A}\right|$, where $v_{0}$ is the volume of a lattice cell. (See explanation in text.)

$+\epsilon_{A A} / 2<\left(V_{A} / \nu_{\perp}\right) \leqslant \epsilon_{A A} / 2$. However, $\Delta H_{1}$ changes sign for a temperature $T$ only if the value of the pressure which corresponds to $\Delta H_{1}=0$ is less than the critical pressure at such temperature.

In order to illustrate such a phenomenology, we shall study the $2 \mathrm{D}$ square lattice case $\left(\nu=4, \nu_{\perp}=1\right)$. In this case, only critical wetting can occur (fluctuation effects preclude any other possibility) and the value of $K_{w}$, at which the transition of critical wetting occurs is given by solution of the equation [29]

$$
\exp \left(2 K_{w}\right)\left[\cosh \left(2 K_{w}\right)-\cosh \left(2 \Delta H_{1}\right)\right]=\sinh \left(2 K_{w}\right) .
$$

Let us first consider the case $\nu_{\perp} \epsilon_{A A} / 2 \leqslant V_{A}<0: \Delta H_{1}<0$ for arbitrary values of $T, z_{B}$, and $V_{B}$. Moreover, $\Delta H_{1}$ becomes more negative as $V_{B}$ decreases, at fixed $T$ and $z_{B}$ (or pressure $p)$. The typical diagrams of complete wetting are displayed in Fig. 3. The region between the wetting transition line and the critical line corresponds to a situation of wetting $s|\beta| \alpha$, and the remaining coexistence region corresponds to a situation of partial wetting. The complete wetting zone increases as $V_{B}$ becomes more negative.

When $\left(V_{A} / \nu_{\perp}\right)<\epsilon_{A A} / 2$, the substrate also favors the adsorption of particles $A$. This determines a competition between $A$ and $B$ species. In general, when $\left(V_{A} / \nu_{\perp}\right)-\epsilon_{A A} / 2$ $<0.1 \epsilon_{A A} \beta$-phase preferential adsorption can only occur if $V_{B}-\left(V_{A} / \nu_{\perp}\right)<\epsilon_{A B}-\epsilon_{A A}$. Figures 4 and 5 display the wetting diagrams for $\epsilon_{A A}<\left(V_{A} / \nu_{\perp}\right)<\epsilon_{A A} / 2$ and $\left(V_{A} / \nu_{\perp}\right)$ $\leqslant \epsilon_{A A}$, respectively. In the first case, the complete wetting transition line originates at $T=0$ and terminates at the temperature of complete wetting for the $A$-pure liquid in the interface substrate- $A$ vapor. For values of $V_{B} \simeq 0, \Delta H_{1}>0$ for all conditions of coexistence, and the configurations are $s|\alpha| \beta$. For $V_{B}<\left(V_{A} / \nu_{\perp}\right)+\epsilon_{A B}-\epsilon_{A A}$, there appear states characterized by $\Delta H_{1}<0$. As a consequence, within the co- 


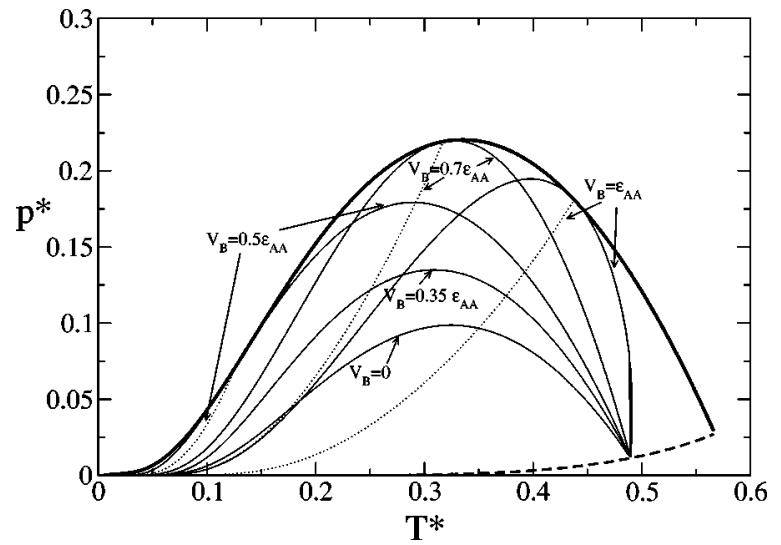

FIG. 4. The same as in Fig. 3 but for $V_{A}=0.75 \epsilon_{A A}$. The dotted lines correspond to the coexistence points at which $\Delta H_{1}=0$. (See text for explanation.)

existence region at the left of the line $p_{n w}(T)$, the substrate adsorbs preferentially particles $B$, and the wetting is of type $s|\beta| \alpha$. On the other hand, at the right of the curve $p_{n w}(T)$, the wetting is of type $s|\alpha| \beta$. The line of wetting transition touches tangentially the critical curve at the point where the curve in turn $p_{n w}(T)$ crosses the critical line. Such a point moves monotonically toward higher temperatures as $V_{B}$ decreases, driving with it the wetting transition line and the $p_{n w}(T)$ curve. As also happened for the $\nu_{\perp} \epsilon_{A A} / 2<V_{A}<0$ case, the wetting transition line from a $s|\beta| \alpha$ complete situation to partial wetting moves to lower pressures, converging toward the $A$-pure liquid-vapor coexistence line for $V_{B} \rightarrow$ $-\infty$ (however, along this line there is a complete wetting by liquid of the vapor-substrate interface over all the temperature range).

When $V_{A} \leqslant \nu_{\perp} \epsilon_{A A}$ (see Fig. 5), for values $V_{B}$ near zero, the coexistence corresponds to complete wetting $s|\alpha| \beta$. Only when $V_{B}<\left(V_{A} / \nu_{\perp}\right)+\epsilon_{A B}-\epsilon_{A A}$ states of partial wetting reappear, near the line $p_{n w}(T)$. The behavior of the latter as well as the wetting transition line is similar to that

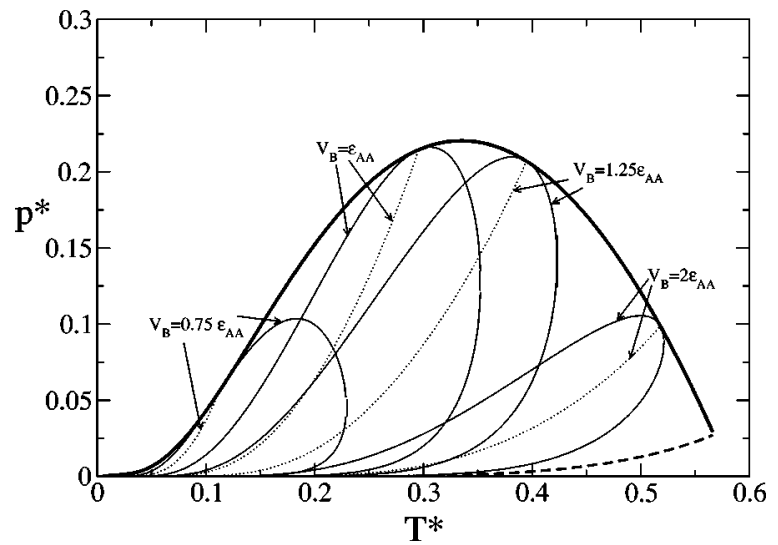

FIG. 5. The same as in Fig. 4 but for $V_{A}=\epsilon_{A A}$. (See text for explanation.)

observed in the previous case, with the difference that the right branch of the wetting transition line (that corresponds to a transition from partial to $s|\alpha| \beta$ complete wetting) begins at zero temperature.

One expects that the behavior of the wetting transition for the three-dimensional case will be qualitatively similar to the two-dimensional case. However, the complete wetting phase diagram for the three-dimensional Ising model is not totally understood yet. Theoretical [30] and simulation studies [31] show, in the case of strictly short-ranged forces and in the absence of an enhanced surface spin-spin coupling, that the wetting transition is continuous only if $K_{w}$ is smaller than $K_{R}$, where $K_{R}$ is the value of the coupling constant corresponding to the roughening transition $\left(K_{R} \approx 1.85 K_{c}\right.$ for the simple cubic lattice). However, if such condition is not fulfilled, the wetting transition will occur through a sequence of first-order layering transitions. Theoretical studies have claimed that the wetting transition can be weakly first order instead of second order [32]. In the present work we will consider the Ising model in the framework of the mean-field Bragg-Williams approximation,

$$
\begin{aligned}
\lim _{N_{\perp} \rightarrow \infty} \frac{1}{N_{\perp}} \ln Z_{\text {Ising }}\left(K, H, \Delta H_{1}\right)= & \Delta H_{1} m_{1}+\sum_{j=1}^{\infty}\left[\frac{\nu_{\|}}{2} K m_{j}^{2}+\nu_{\perp} K m_{j} m_{j+1}+H m_{j}-\left(\frac{1+m_{j}}{2}\right) \ln \left(\frac{1+m_{j}}{2}\right)\right. \\
& \left.-\left(\frac{1-m_{j}}{2}\right) \ln \left(\frac{1-m_{j}}{2}\right)\right],
\end{aligned}
$$

where $N_{\perp}$ is the number of cells in a layer, an $\left\{m_{j}\right\}, j$ $=1,2, \ldots \infty$ is the equilibrium magnetization profile obtained by solving the Euler-Lagrange equations

$$
m_{j}=\tanh \left(\nu_{\|} K m_{j}+\nu_{\perp} K\left(m_{j-1}+m_{j+1}\right)+H\right),
$$

where $m_{0} \equiv \Delta H_{1} / \nu_{\perp} K$. This set of equations must be solved in conjunction with the asymptotic condition $m_{j} \rightarrow m_{b}$ for $j \rightarrow \infty$, where $m_{b}$ is the spontaneous magnetization per site in the bulk case with coupling constant $K$ and magnetic field $H$. In general, different solutions of Eq. (13) are found. The equilibrium profile is the solution that maximizes functional (12). This approach neglects capillary fluctuations, that it is equivalent to assume $K_{R} \equiv K_{c}$ and the wetting transition occurs as a sequence of layering transitions [33,34]. Once the equilibrium magnetization profile is computed, the surface tension $\sigma$ is obtained via 


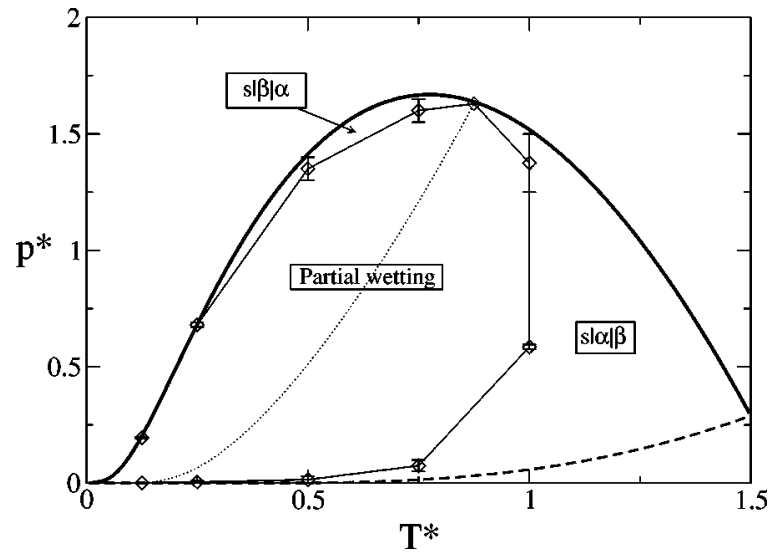

FIG. 6. Wetting phase diagram for the simple cubic (sc) LT model in the mean-field approximation. The substrate-fluid interactions are $V_{A}=\epsilon_{A A}$ and $V_{B}=1.25 \epsilon_{A A}$. The critical line (thick solid), $A$-pure vapor-liquid transition line (dashed), and $p_{n w}(T)$ line (dotted) are plotted. The wetting transition points (diamonds) are also represented. The line that joins them is only to guide the eye.

$$
\sigma=(\Omega+p V) / A,
$$

where $\Omega=-k_{B} T \ln \Xi$, with $\Xi$ defined by Eqs. (1) and (12), and the pressure $p$ by Eq. (5). The adsorption $\Gamma$, that it is the relevant order parameter in the wetting transition, is defined as

$$
\Gamma=\sum_{j=1}^{\infty}\left(X_{A}(j)-X_{A}^{b}\right),
$$

where $X_{A}(j)$ is the $A$ molar fraction profile, Eq. (6), by using the approximation $\left\langle s_{i} s_{j}\right\rangle \approx m_{i} m_{j}$. Finally, $X_{A}^{b}$ is its bulk value.

We have studied the simple cubic lattice, for which $\nu$ $=6$ and $\nu_{\perp}=1$. The magnetization profiles have been obtained by an iterative method of Eq. (13) for $j$ $=1, \ldots, N_{\max }$, imposing the condition $m_{j}=m_{b}$ for $j$ $>N_{\text {max }}$. In order to sample the solution space, we use sharp kink profiles $\left[m_{j}=+1(-1)\right.$ for $\Delta H_{1}>0\left(\Delta H_{1}<0\right)$, respectively, if $j \leqslant j_{0}$ and $m_{j}=m_{b}$ for $j>j_{0}$ ] as initial magnetization profiles. Starting the iteration with different initial profiles, in general, the algorithm converges toward different local minima of the free energy. As already mentioned, the global free energy minimum gives the true equilibrium magnetization profile. Finally, we have considered different values of $N_{\max }$, since the equilibrium profile in a complete wetting situation has a strong size dependence. On the contrary, no appreciable finite width effects occur in a partial wetting situation for $N_{\max }$ large enough. In order to locate the wetting transition, we studied the equilibrium profiles at bulk coexistence $(H=0)$ and constant temperature, varying the pressure.

The wetting phase diagram for $V_{A}=\epsilon_{A A}$ and $V_{B}$ $=1.25 \epsilon_{A A}$ is plotted in Fig. 6. As one can see, the qualitative behavior of the wetting transition is similar to that observed in the two-dimensional case. However, the wetting transition is associated to series of first-order layering transitions be-

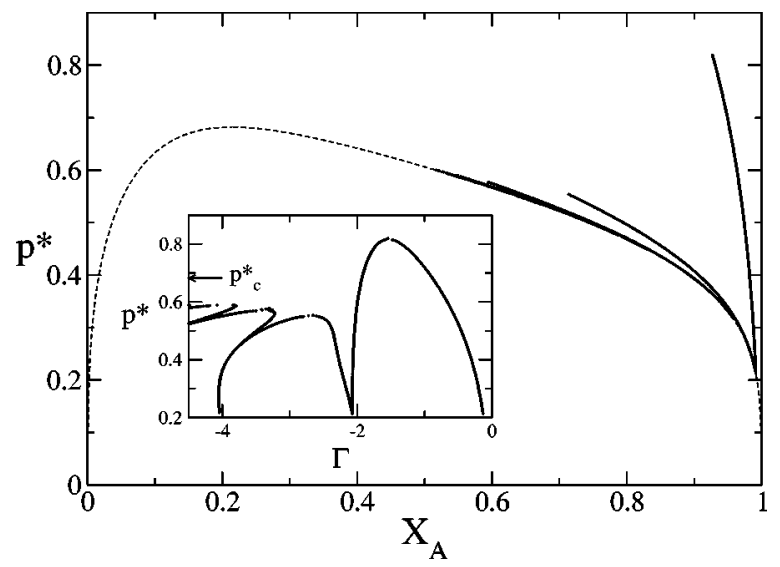

FIG. 7. Layering transitions for $T^{*}=0.25$ for the sc LT model in the mean-field approach. The interaction couplings are the same as in Fig. 6. The dashed lines correspond to the bulk coexistence, and the solid lines correspond to the bulk conditions corresponding to the layering transitions. In the inset, the coexistence adsorptions for the layering transition (only the first four transitions are plotted). The arrow shows the bulk critical pressure.

tween surface phases with the same surface tension, but different adsorptions. The behavior of the layering transitions at low temperatures shows surprising features. Figure 7 shows the first four layering transitions for $T^{*} \equiv k_{B} T /\left|\epsilon_{A A}\right|=0.25$. It is observed that the first layering transition does occur for pressures higher than the bulk critical pressure. Consequently, for a fixed pressure between the first layering transition critical point and the bulk critical point corresponding to this temperature, the layering transition will extend to lower temperatures than the critical temperature corresponding to the LCSP. This behavior is found only for $s|\beta| \alpha$ complete wetting in the low-temperature zone. The pressure is an increasing function on $H$ for fixed temperature $T$ and $z_{B}$ (so both $K$ and $\Delta H_{1}$ are fixed). Consequently, as $K>K_{c}$ for the layering transition critical point, the corresponding pressure can only be bigger than the bulk critical pressure if the transition occurs for $H>0$. This implies that $\Delta H_{1}$ must be negative, and thus the wetting is of type $s|\beta| \alpha$. The physical interpretation of this phenomenon is that the surface adsorbs preferentially $B$ particles. Such a mechanism competes with the $A-B$ bonding (at least close to the substrate). Therefore, the tendency to phase separate in a fluid layer close to the substrate is enhanced (because the $A-B$ bonding favors mixing) for temperatures lower than the (lower) critical temperature. This fact was also observed in computer simulations of a different model of associating binary mixture in Ref. [17]. However, we must stress that this phenomenon is not related to an enhanced coupling (i.e., the interactions between particles in the first layer close to the substrate are increased respect the bulk values), that it is known to stabilize surface transitions to temperatures higher to the bulk critical one in the Ising model [2]. On the other hand, the competition between substrate preferential adsorption and the $A-B$ bonding should be also relevant for prewetting transitions.

\section{CONFINEMENT BETWEEN SYMMETRIC WALLS}

We turn to consider the behavior of the Lin-Taylor model when confined between two identical parallel substrates. 
Confinement in the two-dimensional case corresponds to a quasi-one-dimensional situation. Thus fluctuations preclude the existence of capillary transitions if the interactions are short ranged. Consequently, only the 3D case will be studied. We shall consider a simple cubic lattice within the meanfield approximation. The substrate-fluid interactions are set to $V_{A}=\epsilon_{A A}$ and $V_{B}=1.25 \epsilon_{A A}$, that stabilize the first (surface) layering transition at temperatures lower than the lower critical temperature. We also assume that the separation between plates is $N_{D}$ lattice units. The free energy of the confined mixture is given by Eq. (1), with the mean-field partition function $Z_{\text {Ising }}\left(K, H, \Delta H_{1}\right)$ given by

$$
\begin{aligned}
\lim _{N_{\perp} \rightarrow \infty} \frac{1}{N_{\perp}} \ln Z_{\text {Ising }}\left(K, H, \Delta H_{1}\right)= & \Delta H_{1}\left(m_{1}+m_{N_{D}}\right)+\sum_{j=1}^{N_{D}}\left[\frac{\nu_{\|}}{2} K m_{j}^{2}+\nu_{\perp} K m_{j} m_{j+1}+H m_{j}-\left(\frac{1+m_{j}}{2}\right) \ln \left(\frac{1+m_{j}}{2}\right)\right. \\
& \left.-\left(\frac{1-m_{j}}{2}\right) \ln \left(\frac{1-m_{j}}{2}\right)\right] .
\end{aligned}
$$

The equation for the equilibrium profile is given by Eq. (13) with the boundary condition $N_{\max }=N_{D} / 2$ for $N_{D}$ even, or $\left(N_{D}+1\right) / 2$ for $N_{D}$ odd. The values of $m_{j}$ for $j \geqslant N_{\text {max }}$ are obtained from the symmetry of the problem: i.e., $m_{j}$ $=m_{N_{D}-j+1}$. In addition to the layering transitions (related to the phenomenology observed on the semi-infinite case), the capillary transitions results from the shift of the bulk coexistence under confinement. Near the center of the pore both phases have different magnetizations, in contrast with the layering and prewetting transitions where the profiles differ in a localized surface region and they have the same values far enough from that region. In the Ising model, the shift is always toward higher values of $K$ (i.e., lower effective temperature) and $H$ negative ( $H$ positive) when $\Delta H_{1}$ positive ( $\Delta H_{1}$ negative), respectively.

The cases $N_{D}=1$ and $N_{D}=2$ can be studied analytically since $m_{j}=m$ for all $j$. The bulk conditions, for which the capillary transition takes place are shown in Fig. 8. In spite of the fact that the temperature range for which the capillary transition occurs is reduced, at low temperatures the pressure range is largely increased. Hence, at a given pressure, the LCSP shifts to lower temperatures and can even disappear. This means that for certain values of the pressure range there are no immiscibility islands in the confined system, but the usual bell shaped coexistence line terminating in a UCSP. This is a direct consequence of the directional character of the substrate-particle $B$ interaction. The kink that is observed in the capillary critical line corresponds to the crossing of the $p_{n w}(T)$ line.

For larger values of $N_{D}$, the behavior of the capillary transitions are studied numerically. As $N_{D} \rightarrow \infty$ the capillary critical line must move toward the bulk critical curve. We shall consider the isotherm $T^{*}=0.25$, as a representative case of the low-temperature regime. The excess free energy $\omega$ reads

$$
\omega=(\Omega+p V) / A
$$

and converges to $2 \sigma$ when $N_{D} \rightarrow \infty$, with $\sigma$ defined by Eq. (14). The relevant order parameter for the capillary transitions is the average molar fraction $\bar{X}_{A}$, defined as

$$
\bar{X}_{A} \equiv \frac{1}{N_{D}} \sum_{j=1}^{N_{D}} X_{A}(j)
$$

that converges to the bulk coexistence molar fractions as $N_{D} \rightarrow \infty$.

We have obtained the constant temperature phase diagrams for $T^{*}=0.25$ (see Fig. 9). Various transitions are observed for $N_{D} \geqslant 3$. One of these transitions converges toward the bulk phase transition for large separations and is identified with the true capillary transition. The remaining transitions are characterized by having similar average molar fractions and correspond to the layering transitions observed in the presence of a single substrate. The coverages [as defined by Eq. (15)] corresponding to the coexisting phases in these transitions converge very fast to the semi-infinite values, as expected from their surface nature. However, the number of these transitions depends strongly on $N_{D}$, since the capillary

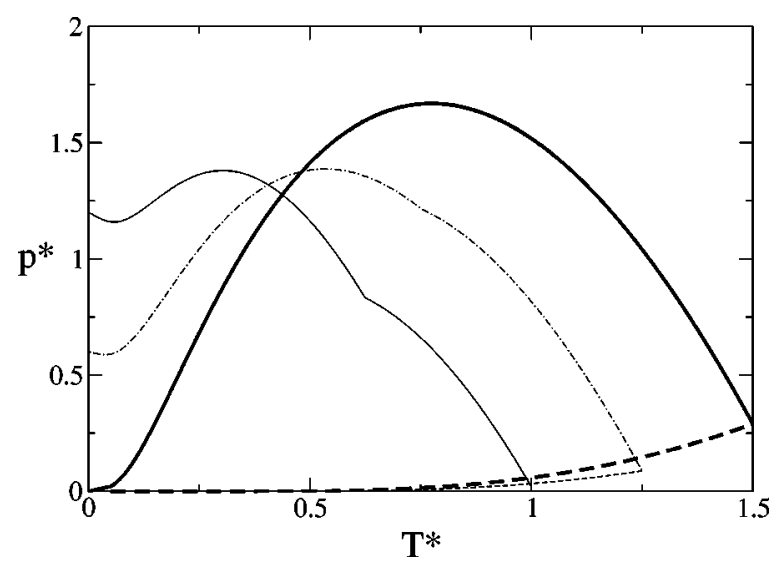

FIG. 8. Capillary transitions of the mean-field sc LT model $\left(V_{A}=\epsilon_{A A}, V_{B}=1.25 \epsilon_{A A}\right)$ for $N_{D}=1$ (thin solid line) and $N_{D}=2$ (dot-dashed line). In both cases, the upper line is the capillary critical line, and the lower line is the $A$-pure capillary gas-liquid transition. The bulk conditions under which there is $\alpha-\beta$ capillary coexistence are the regions enclosed by their respective lines, and the $T=0$ axis. By comparison, the bulk critical line (thick solid line) and the $A$-pure gas-liquid transition line (thick dashed line). 


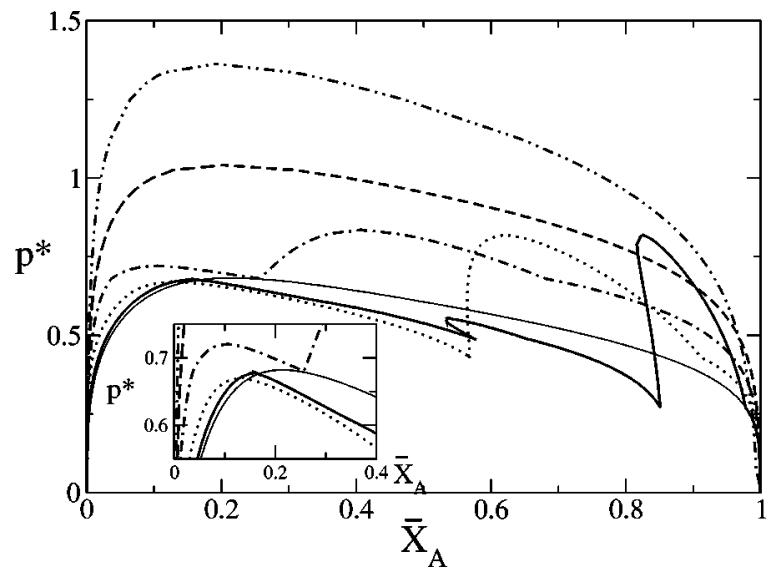

FIG. 9. $\bar{X}_{A}-p$ phase diagrams for $T^{*}=0.25$ and different values of $N_{D}: N_{D}=1$ (dashed-double dotted line), $N_{D}=2$ (dashed line), $N_{D}=5$ (dotted line), and $N_{D}=15$ (thick solid line), under the same conditions as in Fig. 8. The bulk transition (thin solid line) is also plotted for comparison. The inset corresponds to an enlargement of the zone around the bulk critical point.

transition, which depends sensibly on $N_{D}$, competes with them [35,36]. For instance, in the case of $3 \leqslant N_{D}<10$ only the first layering transition appears, while the second appears for $N_{D} \geqslant 10$.

The values of the capillary critical pressure for small $N_{D}$ are larger than the corresponding bulk critical values. By increasing $N_{D}$, the capillary critical pressure decreases up to a minimum, corresponding to a pressure less than the bulk critical pressure. From such value it converges monotonically to the bulk critical value (see Fig. 10). This fact means that along an isobaric the LCSP shifts to lower temperatures only for very narrow pores. Consequently, the stabilizing mechanism of the coexistence region below the bulk LCSP seems to be only effective for small values of $N_{D}$. The deviations of the capillary critical pressure with respect to the bulk value in the range $5<N_{D} \leqslant 15$ follow a power law $\Delta p$ $\sim N_{D}^{-x}$, with $x=1.04 \pm 0.09$. This behavior is consistent with

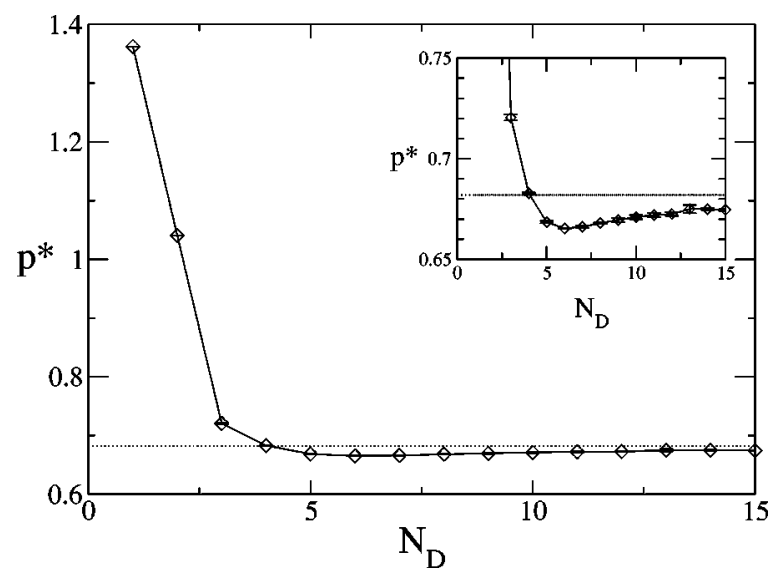

FIG. 10. Capillary reduced critical pressures $p^{*}$ versus $N_{D}$ for $T^{*}=0.25$ (same conditions as in Fig. 8). The dotted line corresponds to the bulk critical pressure. Inset, enlargement of the minimum zone.

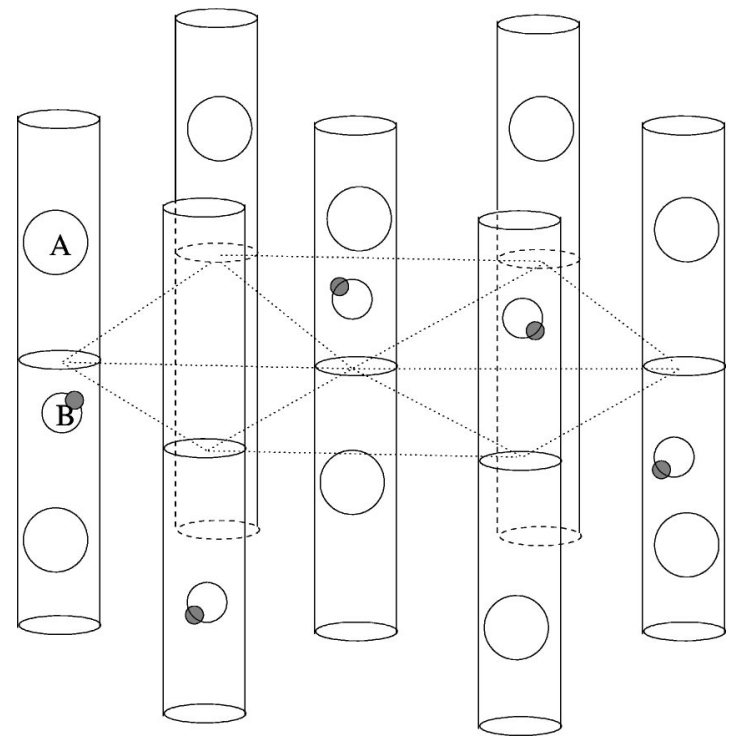

FIG. 11. Schematic representation of the LT model adsorbed in a network of narrow infinitely long cylinders. The $A$ particles are represented as spherical particles, and the $B$ particles as spherical particles with an interaction side (shadow circle). In this case, the coordination number is $\nu_{\perp}=6$.

the findings for the confined lattice gas for intermediate values of $N_{D}$ reported in Ref. [35]. The scaling behavior $\Delta p$ $\sim N_{D}^{-1 / \nu}\left(N_{D}^{-2}\right.$ in the mean-field approach $)$ predicted by the Nakanishi-Fisher theory should be obeyed for larger values of $N_{D}$. However, our results do not extend to that region due to the numerical error in estimating the capillary critical pressure close to the bulk critical point. Nevertheless, we do not expect a change in the tendency for large values of $N_{D}$ and we expect our results to be qualitatively correct for all values of $N_{D}$. It is interesting to note that the slab approximation [35], which corresponds to set $m_{j}=m$ for all $j$, predicts that for sufficiently small $T^{*}$ the capillary critical pressure is larger than the bulk value for all values of $N_{D}$. The failure of this approach is not surprising, since it gives poor results for the confined lattice gas close to the capillary critical point as it overestimates both $K$ and $H$ at the capillary critical point [35]. This fact alters the balance between the different mechanisms involved in the shift of the critical point, leading to the wrong result mentioned above.

\section{THE CYLINDRICAL PORE NETWORK}

Finally, we consider the confinement of the LT mixture in a network of parallel infinitely long, cylindrical pores. This model can be understood as a crude approximation for adsorption in zeolites. It represents another instance in which the substrate can prevent the formation of hydrogen bonds: the geometrical hindrance due to the confinement in very narrow nanopores. We shall assume that the pore diameter is so small that the $A-B$ bonds can form only along the direction $z$ parallel to the cylinder axis (see Fig. 11). The cylinder diameter is taken to be less than twice the $A-B$ collision diameter. In addition, the pores have diameters less than two $A$-particle diameters and they form a two-dimensional net- 
work with coordination number $\nu_{\perp}$. We allow attenuated interactions between particles in nearest-neighbor pores, in order to allow a capillary transition. A similar zeolite model has been employed to study the capillary transition of methane adsorbed in $\mathrm{ALPO}_{4}-5$ [37].

The in-pore fluid-fluid interactions have the same form as in bulk, with coupling constants $\epsilon_{A A}, \epsilon_{A B}$, and $\epsilon_{B B}$. However, the interactions between particles in nearest-neighbor pores are attenuated to $\tilde{\epsilon}_{A A}, \tilde{\epsilon}_{A B}$, and $\tilde{\epsilon}_{B B}\left(\left|\tilde{\epsilon}_{i j}\right|<\left|\epsilon_{i j}\right|\right)$, although the $A-B$ and $B-B$ interactions directional character remains the same as before. This system behaves as a bulk lattice model with anisotropic interactions. The grandcanonical-free energy per unit volume reads

$$
\begin{aligned}
\frac{\Omega}{V}= & -\frac{k_{B} T}{v_{0}}\left[\frac { \nu _ { \perp } } { 2 } \operatorname { l n } \left(1+2 \tilde{z}_{B}+\widetilde{z}_{B}^{2} e^{\left.-\beta \tilde{\epsilon}_{B B}\right)}\right.\right. \\
& +\ln \left(1+2 z_{B}+z_{B}^{2} e^{-\beta \epsilon_{B B}}\right)+H-\frac{\nu_{\perp} K_{\perp}}{2}-K_{\|} \\
& \left.+\frac{1}{N} \ln Z_{\text {Ising }}\left(K_{\perp}, K_{\|}, H\right)\right]
\end{aligned}
$$

where $\tilde{z}_{B} \equiv z_{B} e^{-\beta V_{B}}$ is the effective activity of the $B$ particles oriented in the plane perpendicular to the cylinder axis, and $Z_{\text {Ising }}$ is canonical partition function of an anisotropic Ising model characterized by couplings $K_{\|}$in the parallel directions to the cylinder axis and $K_{\perp}$ in the perpendicular direc- tions, and an effective magnetic field $H . K_{\|}$has the same expression as the bulk coupling constant, Eq. (2), while $K_{\perp}$ and $H$ are defined as

$$
\begin{aligned}
K_{\perp}=- & \frac{\beta \tilde{\epsilon}_{A A}}{4}+\frac{1}{2} \ln \left(\frac{\sqrt{1+2 \tilde{z}_{B}+\tilde{z}_{B}^{2} e^{-\beta \tilde{\epsilon}_{B B}}}}{1+\tilde{z}_{B} e^{-\beta \tilde{\epsilon}_{A B}}}\right), \\
\widetilde{H}= & H+\nu_{\perp}\left[\frac{\beta}{4}\left(\epsilon_{A A}-\tilde{\epsilon}_{A A}-\frac{2 V_{A}}{\nu_{\perp}}\right)\right. \\
& \left.+\frac{1}{4} \ln \left(\frac{1+2 z_{B}+z_{B}^{2} e^{-\beta \epsilon_{B B}}}{1+2 \tilde{z}_{B}+\tilde{z}_{B}^{2} e^{-\beta \tilde{\epsilon}_{B B}}}\right)\right],
\end{aligned}
$$

with $H$ given by Eq. (3). One can see that confinement shifts the effective magnetic field $H$ with respect to its bulk value with a contribution analogous to that found for planar substrates [compare Eqs. (4) and (21)]. In addition, the confinement determines an anisotropy in the couplings. The $A$-particle molar fraction $X_{A}$ reads

$$
X_{A}=\frac{n_{A}}{n_{A}+n_{B}^{1}+n_{B}^{2}},
$$

where $n_{A}=(1+m) / 2 v_{0}$ is the $A$-particle density, and $n_{B}^{1}$ $\left(n_{B}^{2}\right)$ is the density of $B$ particles oriented in a perpendicular (parallel) direction to the cylinder axis, respectively,

$$
\begin{aligned}
n_{B}^{1}= & \frac{\nu_{\perp}}{4 v_{0}}\left(\frac{\tilde{z}_{B}+\widetilde{z}_{B}^{2} e^{-\beta \tilde{\epsilon}_{B B}}}{1+2 \tilde{z}_{B}+\widetilde{z}_{B}^{2} e^{-\beta \tilde{\epsilon}_{B B}}}+\frac{\tilde{z}_{B} e^{-\beta \tilde{\epsilon}_{A B}}}{1+\tilde{z}_{B} e^{-\beta \tilde{\epsilon}_{A B}}}\right)-\frac{\nu_{\perp}}{4 v_{0}}\left(\frac{\tilde{z}_{B}+\widetilde{z}_{B}^{2} e^{-\beta \tilde{\epsilon}_{B B}}}{1+2 \tilde{z}_{B}+\tilde{z}_{B}^{2} e^{-\beta \tilde{\epsilon}_{B B}}}-\frac{\tilde{z}_{B} e^{-\beta \tilde{\epsilon}_{A B}}}{1+\tilde{z}_{B} e^{-\beta \tilde{\epsilon}_{A B}}}\right)\left(\frac{\partial \ln Z_{I s i n g} / N}{\partial K_{\perp}}\right) \\
& -\frac{\nu_{\perp}}{2 v_{0}} \frac{\tilde{z}_{B}+\widetilde{z}_{B}^{2} e^{-\beta \tilde{\epsilon}_{B B}}}{1+2 \tilde{z}_{B}+\tilde{z}_{B}^{2} e^{-\beta \tilde{\epsilon}_{B B}}} m, \\
n_{B}^{2}= & \frac{1}{2 v_{0}}\left(\frac{z_{B}+z_{B}^{2} e^{-\beta \epsilon_{B B}}}{1+2 z_{B}+z_{B}^{2} e^{-\beta \epsilon_{B B}}}+\frac{z_{B} e^{-\beta \epsilon_{A B}}}{1+z_{B} e^{-\beta \epsilon_{A B}}}\right)-\frac{1}{2 v_{0}}\left(\frac{z_{B}+z_{B}^{2} e^{-\beta \epsilon_{B B}}}{1+2 z_{B}+z_{B}^{2} e^{-\beta \epsilon_{B B}}}-\frac{z_{B} e^{-\beta \epsilon_{A B}}}{1+z_{B} e^{-\beta \epsilon_{A B}}}\right)\left(\frac{\partial \ln Z_{I s i n g} / N}{\partial K_{\|}}\right) \\
& -\frac{1}{v_{0}} \frac{z_{B}+z_{B}^{2} e^{-\beta \epsilon_{B B}}}{1+2 z_{B}+z_{B}^{2} e^{-\beta \epsilon_{B B}}} m .
\end{aligned}
$$

Phases characterized by different molar fractions $X_{A}$ may coexist when the spontaneous (i.e., $\widetilde{H}=0$ ) magnetization $m$ $= \pm|m| \neq 0$. We consider the case $\epsilon_{A A}=-1, \epsilon_{A B}=-0.6$, and $\epsilon_{B B}=0$, that corresponds to a case that presents closed loops of immiscibility in the bulk. On the other hand, as the hydrogen bond interactions are very short ranged, we will take $\tilde{\epsilon}_{A B}=\tilde{\epsilon}_{B B}=0$ as physically sensible values. Moreover, $\tilde{\epsilon}_{A A}$ is set to be $\alpha \epsilon_{A A}$, where $\alpha$ is a positive number less than one.

In order to discriminate between different mechanisms, we chose the substrate-fluid interactions in such a way that
$\widetilde{H}=H$, i.e., $V_{A}=\nu_{\perp}(1-\alpha) \epsilon_{A A} / 2$ and $V_{B}=0$. Hence, the preferential substrate adsorption effect (very similar to that studied in the slab geometry) is suppressed and we can focus on the coupling anisotropy.

We have studied the 2D square lattice case, for which analytical expressions for $Z_{D, \nu}$ are known in the anisotropic case [38]. However, we expect that the features obtained for this case will be qualitatively correct also for the 3D case. From the exact solution, the values of $\left(K_{\perp}, K_{\|}\right)$that correspond to the phase coexistence satisfy the following relationship: 


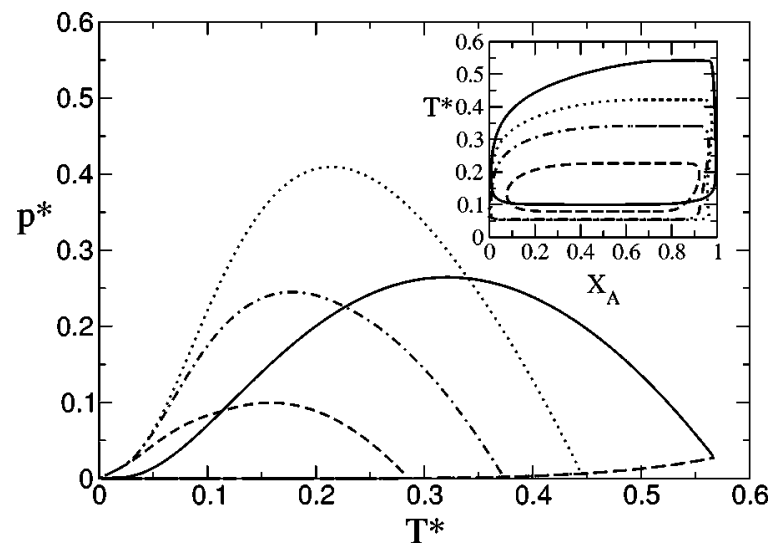

FIG. 12. Capillary transitions in the network of cylindrical pores. The reservoir conditions corresponding to the capillary critical lines are represented for different values of $\alpha: \alpha=0.2$ (dashed line), $\alpha=0.4$ (dotted-dashed line), and $\alpha=0.6$ (dotted line). The bulk critical line (solid line) and the $A$-pure vapor-liquid line (longdashed line) are also plotted. In the inset, the phase diagrams corresponding to $p^{*}=0.07$ for the same values of $\alpha$ (the symbol meaning is the same as previously) and their comparison with the bulk phase diagram (solid line).

$$
\sinh \left(2 K_{\|}\right) \sinh \left(2 K_{\perp}\right) \geqslant 1,
$$

where equality holds only at the critical point. It is clear from such an expression that, even for $K_{\|}$(equal to the bulk value) less than the isotropic bulk value, the system can undergo a phase transition for $K_{\perp}$ large enough. Note that since $K_{\perp}=$ $-\beta \alpha \epsilon_{A A} / 4$, this condition is fulfilled in the low-temperature region. This fact is also observed in the mean-field approach, for which the coexistence condition is $\nu_{\perp} K_{\perp}+2 K_{\|} \geqslant 1$.

Figure 12 shows the reservoir conditions under which the capillary phase transition occurs for different values of $\alpha$. There also exist immiscibility islands for the capillary transition within the porous medium [see inset in Fig. 12], but the corresponding LCSP occurs at temperatures lower than those of the bulk LCSP at the same pressure. As $\alpha$ increases the temperature and pressure range for which one observes capillary transition increases. Hence, the immiscibility islands become larger with $\alpha$, but mainly in the hightemperature region. In fact, the temperature corresponding to the LCSP is rather insensitive to the value of $\alpha$, and it is always below the temperature corresponding to the bulk LCSP. Our findings can be understood by a simple physical argument. The geometry forbids the formation of hydrogen bondings in the directions perpendicular to the cylinder axis. So, the confinement effectively reduces the $A-B$ bonds re- spect to the bulk case, and consequently the phase separation is enhanced in the lower-temperature regime, in complete agreement with our results.

\section{DISCUSSION AND CONCLUSIONS}

In this paper we have studied the effect on the phase equilibria of an associating binary mixture in the presence of substrates and under confinement in very simple geometries. We have focused on the conditions in which bulk system exhibits closed loops of immiscibility. Our results show clearly that the presence of directional fluid-substrate interactions can stabilize purely surface transitions (such as layering and prewetting) and also for the phase separation under confinement in nanopores, for temperatures below the bulk LCSP. However, this mechanism becomes inefficient for the capillary transition when the surface-to-volume ratio is small, and consequently we doubt that it can explain the experimental results in Refs. [18,19], which would correspond to the latter case. The disagreement with the scaling predictions in the cases, in which the temperature shift depends on the substrate remains, in any case, unsolved. The experimental data show a capillary critical temperature dependence on the film width that it is not consistent with the scaling predictions $[4,5]$, and it could be a signature of an emerging new characteristic length scale. However, to our knowledge there is no theoretical explanation of such a behavior.

Some final remarks are pertinent. First, the present model represents a simplified description of an associating binary mixture, but keeps all the physical ingredients needed to reproduce the correct phenomenology. Consequently, we are confident that our conclusions can be relevant also for more sophisticated models. Second, fluid adsorption in nanotubes and zeolites is experimentally feasible and they could provide experimental probes of our theoretical predictions.

\section{ACKNOWLEDGMENTS}

The authors wish to thank Dr. I. Rodríguez-Ponce, Professor G. Jackson, and Professor A. O. Parry for their interest and useful comments on this paper. J.M.R.-E. and L.F.R. gratefully acknowledge financial support for this research by Grant No. PB97-0712 from DGICyT (Spain) and Grant No. FQM-205 from PAI (Junta de Andalucía). J.M.R.-E. also wishes to thank the Ministerio de Educación, Cultura y Deporte (Spain) for partial financial support. Finally U.M.B.M acknowledges financial support by Ministero dell'Istruzione, dell'Universitá e della Ricerca, Cofin 2001 Prot. No. 2001023848.
[1] R. Evans, in Fundamentals of Inhomogeneous Fluids, edited by D. Henderson (Marcel Dekker, New York, 1992).

[2] H. Nakanishi and M.E. Fisher, Phys. Rev. Lett. 49, 1565 (1982).

[3] M.E. Fisher and H. Nakanishi, J. Chem. Phys. 75, 5857 (1981).
[4] H. Nakanishi and M.E. Fisher, J. Chem. Phys. 78, 3279 (1983).

[5] H. Nakanishi and M.E. Fisher, J. Phys. C 16, L95 (1983).

[6] R. Evans, U. Marini Bettolo Marconi and P. Tarazona, J. Chem. Phys. 84, 2376 (1986).

[7] R. Evans and U. Marini Bettolo Marconi, J. Chem. Phys. 86, 7138 (1987). 
[8] P. Tarazona, R. Evans, and U. Marini Bettolo Marconi, Mol. Phys. 60, 573 (1987).

[9] C.S. Hudson, Z. Phys. Chem. (Munich) 47, 113 (1904).

[10] J. Hirschfelder, D. Stevenson, and H. Eyring, J. Chem. Phys. 5, 896 (1937).

[11] J.A. Barker and W. Fock, Discuss. Faraday Soc. 15, 188 (1953).

[12] J.C. Wheeler, J. Chem. Phys. 62, 433 (1975).

[13] J.S. Walker and C.A. Vause, Phys. Lett. 79A, 421 (1980).

[14] G. Jackson, Mol. Phys. 72, 1365 (1991).

[15] L.A. Davies, G. Jackson, and L.F. Rull, Phys. Rev. Lett. 82, 5285 (1999).

[16] L.A. Davies, G. Jackson, and L.F. Rull, Phys. Rev. E 61, 2245 (2000).

[17] M. Kotelyanskii and S.K. Kumar, Phys. Rev. Lett. 80, 1252 (1998).

[18] B.A. Scheibner, M.R. Meadows, R.C. Mockler, and W.J. O'Sullivan, Phys. Rev. Lett. 43, 590 (1979).

[19] M.R. Meadows, B.A. Scheibner, R.C. Mockler, and W.J. O’Sullivan, Phys. Rev. Lett. 43, 592 (1979).

[20] J.C. Lin and P.L. Taylor, Phys. Rev. E 49, 2058 (1994).

[21] J.C. Lin and P.L. Taylor, Phys. Rev. Lett. 73, 2863 (1994).

[22] J.M. Romero-Enrique, I. Rodríguez Ponce, L.F. Rull, and U. Marini Bettolo Marconi, Phys. Rev. Lett. 79, 3543 (1997).

[23] J.M. Romero-Enrique, I. Rodríguez Ponce, L.F. Rull, and U. Marini Bettolo Marconi, Mol. Phys. 93, 501 (1998).

[24] J.M. Romero-Enrique, I. Rodríguez Ponce, L.F. Rull, and U.
Marini Bettolo Marconi, Mol. Phys. 95, 571 (1998).

[25] J.M. Romero-Enrique, I. Rodríguez Ponce, L.F. Rull, and U. Marini Bettolo Marconi, Phys. Chem. Chem. Phys. 1, 4271 (1999).

[26] For a general review of wetting, see for example, S. Dietrich, in Phase Transitions and Critical Phenomena, edited by C. Domb and J.L. Lebowitz (Academic Press, New York, 1988), Vol. 12, p. 1.

[27] J.S. Rowlinson and B. Widom, Molecular Theory of Capillary (Clarendon Press, Oxford, 1982).

[28] G. Forgacs, R. Lipowsky, and Th.M. Nieuwenhuizen, in Phase Transitions and Critical Phenomena, edited by C. Domb and J.L. Lebowitz (Academic Press, New York, 1991), Vol. 14, p. 135.

[29] D.B. Abraham, Phys. Rev. Lett. 44, 1165 (1980).

[30] R. Pandit, M. Schick, and M. Wortis, Phys. Rev. B 26, 5112 (1982).

[31] K. Binder and D.P. Landau, Phys. Rev. B 37, 1745 (1988).

[32] M.E. Fisher and A.J. Jin, Phys. Rev. Lett. 69, 792 (1992).

[33] M.J. de Oliveira and R.B. Griffiths, Surf. Sci. 71, 687 (1978).

[34] C. Ebner, Phys. Rev. A 22, 2776 (1980).

[35] E. Bruno, U. Marini Bettolo Marconi, and R. Evans, Physica A 141, 187 (1987).

[36] R. Evans and P. Tarazona, Phys. Rev. Lett. 52, 557 (1984).

[37] R. Radhakrishnan and K.E. Gubbins, Phys. Rev. Lett. 79, 2847 (1997).

[38] L. Onsager, Phys. Rev. 65, 117 (1944). 